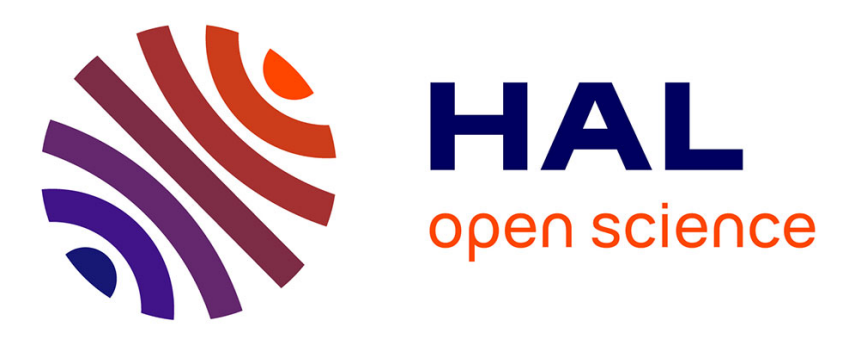

\title{
Increase of Singularity-Free Zones in the Workspace of Parallel Manipulators Using Mechanisms of Variable Structure
}

\author{
Vigen Arakelian, Sébastien Briot, Victor Glazunov
}

\section{- To cite this version:}

Vigen Arakelian, Sébastien Briot, Victor Glazunov. Increase of Singularity-Free Zones in the Workspace of Parallel Manipulators Using Mechanisms of Variable Structure. Mechanism and Machine Theory, 2008, 43 (9), pp.1129-1140. hal-00451884

\section{HAL Id: hal-00451884 https://hal.science/hal-00451884}

Submitted on 25 Jun 2019

HAL is a multi-disciplinary open access archive for the deposit and dissemination of scientific research documents, whether they are published or not. The documents may come from teaching and research institutions in France or abroad, or from public or private research centers.
L'archive ouverte pluridisciplinaire HAL, est destinée au dépôt et à la diffusion de documents scientifiques de niveau recherche, publiés ou non, émanant des établissements d'enseignement et de recherche français ou étrangers, des laboratoires publics ou privés. 


\title{
Increase of singularity-free zones in the workspace of parallel manipulators using mechanisms of variable structure
}

\author{
Vigen Arakelian ${ }^{\text {a) }}$, Sébastien Briot ${ }^{\text {a) }}$, Victor Glazunov ${ }^{\text {b) }}$ \\ a) Département de Génie Mécanique et Automatique - L.G.C.G.M. EA3913 \\ Institut National des Sciences Appliquées (I.N.S.A.) \\ 20 avenue des Buttes de Coësmes - CS 14315 \\ F-35043 Rennes, France \\ E-mails : vigen.arakelyan@insa-rennes.fr \\ sebastien.briot@ens.insa-rennes.fr \\ b) Mechanical Engineering Research Institute \\ Russian Academy of Sciences \\ M.Kharitonyevski str. 4, \\ 101990 Moscow, Russia \\ E-mail:vaglznv@mail.ru
}

\begin{abstract}
This paper is focused on the study of singularity of planar parallel manipulators taking into account the force transmission, i.e. study of singularity of planar manipulator by introducing the force transmission factor. Thus the singularity zones in the workspace of the manipulator are defined not only by kinematic criterions from the theoretical perfect model of the manipulator but also by the quality of force transmission. For this purpose, the pressure angle is used as an indicator of force transmission. The optimal control of the pressure angle for a given trajectory of the manipulator is realized by means of legs with variable structure. The suggested procedure to determination of the optimal structure of the planar parallel manipulator 3-RPR is illustrated by two numerical simulations.
\end{abstract}

Keywords: Parallel manipulator, singularity-free zones, pressure angle, force transmissivity analysis

\section{Introduction}

It is well-known that the closed-loop of parallel manipulators limits the motion of the platform and creates special singular zones inside the workspace [1]. The workspace of the parallel manipulators which is less than the serial manipulators becomes smaller and limits their functional performance.

One of the most evident solutions of this problem is the introduction in the initial system of complementary actuators, which make it possible to eliminate the singular configurations of the parallel manipulator by means of optimal control of the motion [2,3]. However, it is an expensive solution to the problem because of the additional actuators and the complicated control of the manipulator caused by actuation redundancy.

In this paper we propose a new solution, which carried out by using mechanisms of variable structure, i.e. a mechanism whose structure parameters can be altered. With regard to the determination of singularity-free zones inside the workspace we propose a kinetostatic approach taking account of the force transmission.

The physical interpretation of a singularity in kinematics refers to those configurations in which the number of degrees of freedom of the mechanical structure changes instantaneously, either the manipulator gains some additional, uncontrollable degrees of freedom or loses some degrees of freedom. Algebraically, a singularity analysis is based on the properties of the 
Jacobian matrices of the mechanical structure, i.e. when the Jacobian matrices relating the input speeds and the output speeds become rank deficient [4-16]. However, it is also wellknown that when the parallel manipulator is close to a singular configuration, it loses the stiffness and the quality of motion transmission, as a result, the payload capability. Thus, the singularity zones must be avoided and an indicator of the quality of motion transmission close to the singular configurations of parallel manipulators must be defined. In the present work we use a kinetostatic approach for the evaluation of the quality of motion transmission by using the pressure angle, well-known in the mechanism design but not so often applied to the parallel mechanisms. One defines the pressure angle as an angle between vectors of force and velocity of a point at which the force is applied. Thus for best force transmission it would be desirable if the pressure angle will be close to zero. One also knows the transmission angle, which is $90^{\circ}$ minus pressure angle and accordingly should be desirable if it will be close to $90^{\circ}$.

S. Balli and S. Chand [17] considered several examples for determination of transmission angle of planar and spatial mechanisms, particularly, for mechanisms with two degrees of freedom. G. Sutherland and B. Roth [18] showed that the input link of a spatial mechanism tends to move the output link when the transmission wrench is not reciprocal to the output link twist. On the base of this consideration a general index of motion transmission for spatial mechanisms is proposed. The quality of motion and force transmission was successfully summarized in the work of G. Sutherland [19] and C.-C. Lin, W.-T. Chang [20]. The study of G. Sutherland and B. Roth [18] was generalized for any spatial single-loop mechanism in the recent study C. Chen and J. Angeles [21]. O. Alba-Gomez, P. Wenger and A. Pamanes [22] have evaluated the quality of motion in the three degrees of freedom manipulators by means of a kinetostatic indicator, which is similar to the pressure angle. Among several works may be distinguished also the study of J. Lee, J. Duffy and J. Keler, J. [23].

The singularity determined from classical approach taking into account only kinematic aspects give information about some singular positions in the geometrical sense. However, in this case, there is not any information about the zones close to these positions, in which the manipulator loses the quality of motion. In this paper the pressure angle are used for determination of these zones close to the singular positions, which cannot be reached by manipulator.

The singularity analysis can be executed on the base of velocity equations. The left hand side of these equations represents the reciprocal screw products of wrenches acting on the moving platform from kinematic chains and the twist of motion of the platform. The right hand side represents the reciprocal screw products of the same wrenches and the twists corresponding to the actuated kinematic pairs. The singularity of type one exists if all the twists of one of the kinematic chains are linear dependent. The singularity of type two exists if the wrenches acting on the platform from the kinematic chains are linear dependent. These singularities can be detected if the determinants consisting of the Plücker coordinates of mentioned twists (the type one singularity) or of mentioned wrenches (the type two singularity) are equal to zero [15]. But from the point of view of force transmission invalid configuration can appear even when the determinant consisting of the Plücker coordinates of the wrenches acting on the moving platform from the kinematic chains is not equal to zero. That is why we consider $m$ pressure angles (where $m$ is the degree of freedom) and choose the worst of these angles as the criteria of closeness to singularity.

In the present study, we use the pressure angle as an indicator of the quality of motion transmission, and in our opinion such a kinetostatic approach shows the nature of the inaccessibility of parallel manipulators' singular zones better than the kinematic approach. 


\section{The quality of motion transmission and pressure angle}

Let us consider a planar parallel manipulator (Figure 1), which consists of the base, the output link (the moving platform) and three kinematic chains with two revolute pairs $A_{i}, B_{i}$ and one prismatic pair $C_{i}(i=1, \ldots, 3)$. Thus, such a manipulator with three actuators (rotating or linear) has three degrees of freedom. The moving platform can translate in the $x y$ plane and rotate (angle $\phi$ ) with respect to an axis perpendicular to the $x y$ plane. The workspace of the manipulator can be defined as the totality of positions that a moving platform can reach. However, these accessible positions are limited not only by geometrical parameters and the type of actuation of the parallel mechanism but also by force transmission. Especially in the configurations close to the singular positions the force transmission becomes unfavorable and the transmission of motion can be disrupted, as a result, leads to a breakdown of the parallel mechanism or an undesirable motion. The pressure angle is an important criterion for the analysis of the inaccessible zone of parallel manipulators.

However, when the number of links and number of degrees of freedom increase, the determination of the pressure angles becomes more complicated. Let us examine the pressure angles of the considered manipulator. Let's consider that the revolute pairs $A_{i}$ is actuated and passive joints are located at $B_{i}$ and $C_{i}$.

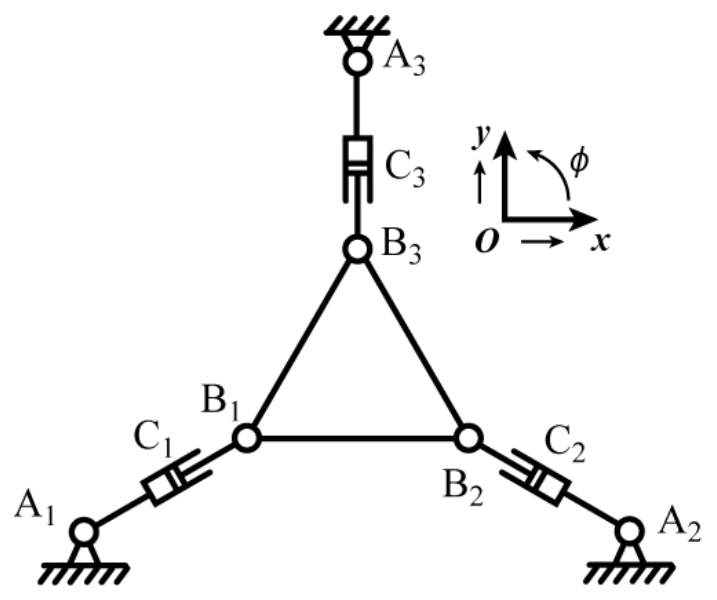

Fig. 1. Planar parallel manipulator 3-RPR.

Thus, each kinematic chain includes one actuated and two passive pairs. The wrench acting to the output link is reciprocal to the unit vectors situated along the axes of nonactuated pairs. Let $\mathbf{E}_{i 1}, \mathbf{E}_{i 2}, \mathbf{E}_{i 3}$ (Figure 2) be the unit vectors of the axes of kinematic pairs, where $i(i=1,2,3)$ is the number of the chain.

Here $\mathbf{E}_{i 1}$ corresponds to rotating actuated pair, $\mathbf{E}_{i 2}$ and $\mathbf{E}_{i 3}$ correspond to sliding and rotating passive pairs accordingly $\left(\mathbf{E}_{i 1}\right.$ and $\mathbf{E}_{i 3}$ directed perpendicular to the plane of the mechanism). These unit screws in any position of the mechanism have Plücker coordinates: $\mathbf{E}_{i 1}\left(\begin{array}{llllll}0 & 0 & 1 & e_{i 1 x}^{0} & e_{i 1 y}^{0} & 0\end{array}\right), \quad \mathbf{E}_{i 2}\left(\begin{array}{llllll}0 & 0 & 0 & e_{i 2 x}^{0} & e_{i 2 y}^{0} & 0\end{array}\right), \quad \mathbf{E}_{i 3}\left(\begin{array}{llllll}0 & 0 & 1 & e_{i 3 x}^{0} & e_{i 3 y}^{0} & 0\end{array}\right)$, where $e_{i 1 x}^{0}=y_{A i}, e_{i 1 y}^{0}=-x_{A i}, e_{i 2 x}^{0}=\left(x_{B i}-x_{A i}\right) / l_{i}, e_{i 2 y}^{0}=\left(y_{B i}-y_{A i}\right) / l_{i}, e_{i 3 x}^{0}=y_{B i}, e_{i 3 y}^{0}=-x_{B i}$, $x_{A i}, x_{B i}, y_{A i}, y_{B i}$ are the coordinates of the point $A_{i}$ and $B_{i}, l_{i}$ is the distance between the points $A_{i}$ and $B_{i}(i=1,2,3)$. 


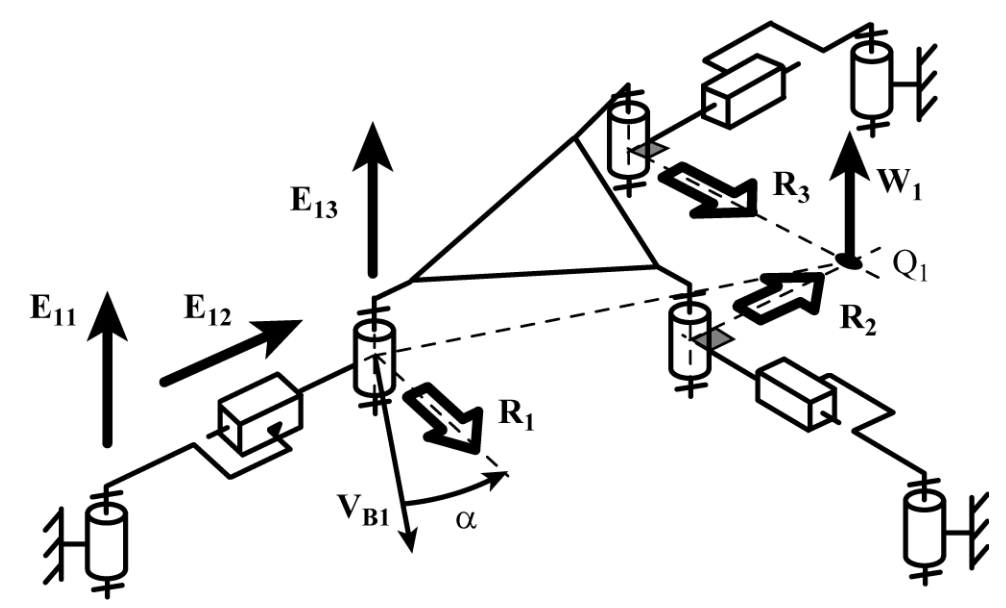

Fig. 2. Representation of the planar parallel manipulator 3-ㅁP in 3D.

For planar mechanisms 3-entries screws can be used [24]. The Plücker coordinates of the unit screws can be described in the matrix $(\mathbf{E})_{i}(i=1,2,3)$ :

$$
(\mathbf{E})_{i}=\left(\begin{array}{ccc}
1 & e^{\circ} & e^{\circ}{ }_{i 1 y} \\
0 & e^{\circ}{ }_{i 2 z} & e^{\circ}{ }_{i 2 y} \\
1 & e^{\circ}{ }_{i 3 z} & e^{\circ}{ }_{i 3 y}
\end{array}\right)
$$

The determinant of the matrix (E) vanishes if the axes $\mathbf{E}_{i 1}$ and $\mathbf{E}_{i 3}$ coincide. It means the occurrence of singularity when the actuator causes only rotation in the joint $\mathbf{E}_{i 3}$.

We can obtain the wrenches $\mathbf{R}_{i}(i=1,2,3)$, which are reciprocal to the unit vectors of the axes of the passive kinematic pairs [24]. They can be written as: $\mathbf{R}_{i}\left(\begin{array}{llllll}r_{i x} & r_{i y} & 0 & 0 & 0 & r_{i z}^{0}\end{array}\right)(i$ $=1,2,3)$. The conditions of reciprocity are:

$$
e_{i 2 x}^{0} r_{i x}+e_{i 2 y}^{0} r_{i y}=0 ; \quad e_{i 3 x}^{0} r_{i x}+e_{i 3 y}^{0} r_{i y}+r_{i z}^{0}=0
$$

The equation (1) means that each connecting kinematic chain determines one wrench of zero pitch (vector). It is perpendicular to the axis $\mathbf{E}_{i 2}$ and intersects the point $B_{i}$. The coordinates of wrenches in the form of the matrix $(\mathbf{R})$ is given by:

$$
(\mathbf{R})=\left(\begin{array}{lll}
r_{1 x} & r_{1 y} & r_{1 z}^{\circ} \\
r_{2 x} & r_{2 y} & r_{2 z}^{\circ} \\
r_{3 x} & r_{3 y} & r_{3 z}^{\circ}
\end{array}\right)
$$

In singular configurations the system of the wrenches $\mathbf{R}_{i}$ degenerates and they intersect in the same point or are parallel [25]. It can be shown by the representation of the components of this matrix. If all the wrenches are parallel then the first two columns are proportional. If all the wrenches intersect in the same point $W\left(\begin{array}{lll}x_{W} & y_{W} & 0\end{array}\right)$ then the coordinate $r_{i z}^{0}(i=1,2,3)$ can be written as $r_{i z}^{0}=r_{1 x} y_{W}-r_{1 y} x_{W}$. In this case in the matrix $(\mathbf{R})$ the third column is a linear combination of the first and seconds columns.

To find the pressure angle we consider the wrenches $\mathbf{R}_{i}$ and the directions of the velocities of the points $B_{i}$ determined by the twists reciprocal to these wrenches. The velocity of the 
point $B_{1}$ is determined by two wrenches $\mathbf{R}_{2}$ and $\mathbf{R}_{3}$. One can find the twist $\mathbf{W}_{1}\left(\begin{array}{llllll}0 & 0 & w_{1 z} & v_{1 x} & v_{1 y} & 0\end{array}\right)$ reciprocal to the wrenches $\mathbf{R}_{2}$ and $\mathbf{R}_{3}$ using the equations:

$$
v_{1 x} r_{2 x}+v_{1 y} r_{2 y}+w_{1 z} r_{2 z}^{0}=0 ; v_{1 x} r_{3 x}+v_{1 y} r_{3 y}+w_{1 z} r_{3 z}^{0}=0
$$

It is obvious that the axis of the twist $\mathbf{W}_{1}$ is situated perpendicular to the plane of the mechanism and intersects the center $Q_{1}$ of velocities of the platform according to the wrenches $\mathbf{R}_{2}$ and $\mathbf{R}_{3}$ (Figure 2). Without interruption of generality the twist $\mathbf{W}_{1}$ can be expressed as $\left(\begin{array}{llllll}0 & 0 & 1 & y_{Q 1} & -x_{Q 1} & 0\end{array}\right)$. The velocity $\mathbf{V}_{\mathrm{B} 1}$ has the coordinates $v_{x B 1}=v_{1 x}-w_{1 z} y_{B 1}=y_{Q 1}-y_{B 1}, v_{y B 1}=v_{1 y}+w_{1 z} x_{B 1}=-x_{Q 1}+x_{B 1}$. If the wrenches $\mathbf{R}_{2}$ and $\mathbf{R}_{3}$ are parallel $\left(r_{2 x}=r_{3 x}, r_{2 y}=r_{3 y}, r_{2 z}^{0} \neq r_{3 z}^{0}\right)$ then $w_{1 z}=0$ and $\mathbf{V}_{\mathrm{B} 1}$ is perpendicular to these wrenches $\mathbf{R}_{2}$ and $\mathbf{R}_{3}$. Finally, the pressure angle can be written as:

$$
\alpha_{1}=\left|\arccos \left(\mathbf{V}_{B 1} \mathbf{R}_{1} /\left|\mathbf{V}_{B 1} \| \mathbf{R}_{1}\right|\right)\right|
$$

It was noted that in the singular configurations all the pressure angles are equal to $90^{\circ}$. Indeed, in this case the axis of the wrench $\mathbf{R}_{1}$ intersects the axes of the wrenches $\mathbf{R}_{2}$ and $\mathbf{R}_{3}$ and the velocity $\mathbf{V}_{\mathrm{B} 1}$ is perpendicular to the axis of the wrench $\mathbf{R}_{1}$.

Thus, the pressure angles can be determined at the joints of each kinematic chain then the maximum value of the pressure angles can be compared with their limit values. In this way we have mapped whole workspace of the parallel manipulator to detect the inaccessible zones with unfavourable values of the pressure angles.

It should be noted that the singularity analysis can be executed on the base of velocity equations. The left hand side of these equations represents the reciprocal screw products of wrenches acting on the moving platform from kinematic chains and the twist of motion of the platform. The right hand side represents the reciprocal screw products of the same wrenches and the twists corresponding to the actuated kinematic pairs. The singularity of type one exists if all the twists of one of the kinematic chains are linear dependent. The singularity of type two exists if the wrenches acting on the platform from the kinematic chains are linear dependent. These singularities can be detected if the determinants consisting of the Plücker coordinates of mentioned twists (the type one singularity) or of mentioned wrenches (the type two singularity) are equal to zero [15]. But from the point of view of force transmission invalid configuration can appear even when the determinant consisting of the Plücker coordinates of the wrenches acting on the moving platform from the kinematic chains is not equal to zero. That is why we consider $m$ pressure angles (where $m$ is the degree of freedom) and choose the worst of these angles as the criteria of closeness to singularity. Thus the standard screw method allows the determination of singular positions only in geometrical sense. By using the pressure angle we determine the volumes, in which the force transmission ability is invalid.

If the prescribed path of the parallel manipulator intersects any unacceptable zone in which the pressure angle has an inadmissible value the transmission of the motion can be disrupted. In this case, it is necessary to change the structural parameters of the mechanism, i.e. the input motions. It will be shown in the following section.

\section{Legs with variable structure}

Figure 3 shows a schematic of the modified leg with the added articulated dyad. The rotating actuators are mounted on the base and connected by electromagnetic clutches with 
the links $A_{i} D_{i}$ and $A_{i} C_{i}$. These two input links cannot actuate simultaneously and the input motion can be transmitted either by the link $A_{i} D_{i}$ or $A_{i} C_{i}(i=1,2,3)$. In this way we can obtain the leg of the mechanisms with different structural parameters, which allow increasing the singularity-free zones in the workspace of the considered parallel manipulator.

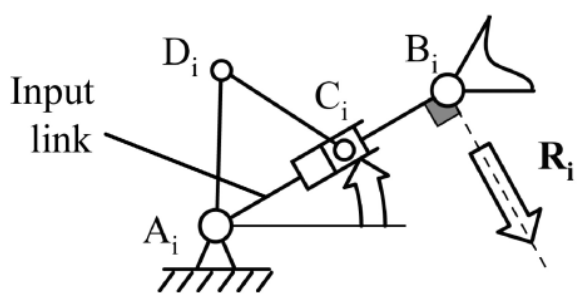

a)

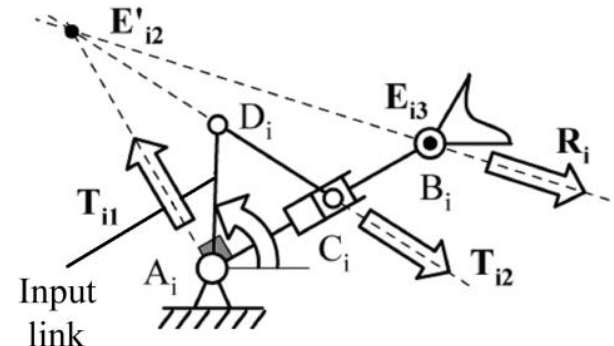

b)

Fig. 3. Leg with variable structure: a) input link $A_{i} C_{i}$; b) input link $A_{i} D_{i}$.

By example, one or all of the pairs $A_{i}$ (Figure 1) can be passive and the prismatic pairs $(i=$ $1,2,3)$ can be actuated by the chain $A_{i} D_{i} C_{i}$. In this case, the actuator torque is transmitted to the link $A_{i} D_{i}$, which becomes an input link and moves the prismatic pair.

It should be noted that the mobility of the modified manipulator is not changed and it is always equal to three.

Let us consider the system of wrenches existing in this case. In the previous case by fixed actuator the link $B_{i} C_{i}$ had translation mobility along the axis of the prismatic pair. In this case the link $B_{i} C_{i}$ is constrained by two wrenches of zero pitch $\mathbf{T}_{i 1}$ and $\mathbf{T}_{i 2}$. The wrench $\mathbf{T}_{i 1}$ is reciprocal to the unit screws of the axes of two kinematic pairs. One of them is rotating and its axis intersects the plane of the mechanism in the point $A_{i}$. The second of them is prismatic and its axis is directed along the line $A_{i} B_{\mathrm{i}}$. The Plücker coordinates of the unit screws of the axes of these kinematic pairs are the same as the coordinates of $\mathbf{E}_{i 1}$ and $\mathbf{E}_{i 2}$ of the previous case. Therefore the axis of the wrench $\mathbf{T}_{i 1}$ is perpendicular to the line $A_{i} B_{i}$ and intersects the point $A_{i}$.

The wrench $\mathbf{T}_{i 2}$ is reciprocal to the unit screws of the axes of two rotating kinematic pairs $C_{i}$ and $D_{i}$. Therefore the axis of the wrench $\mathbf{T}_{i 2}$ coincides with the axis of the link $C_{i} D_{i}$.

The unit screw $\mathbf{E}_{i 2}^{\prime}\left(\begin{array}{llllll}0 & 0 & 1 & e_{i 2 x}^{0^{\prime}} & e_{i 2 y}^{0^{\prime}} & 0\end{array}\right)$ of the twist of the link $B_{i} C_{i}$ is reciprocal to the wrenches $\mathbf{T}_{i 1}$ and $\mathbf{T}_{i 2}$. This twist is of zero pitch and is perpendicular to the plane of the mechanism. Corresponding to this the point of intersection of the wrenches $\mathbf{T}_{i 1}$ and $\mathbf{T}_{i 2}$ coincides with the point of intersection of the axis $\mathbf{E}^{\boldsymbol{X}_{i 2}}$ and the plane of mechanism. If the link $C_{i} D_{i}$ is perpendicular to the link $B_{i} C_{i}$ then the wrenches $\mathbf{T}_{i 1}$ and $\mathbf{T}_{i 2}$ are parallel and the instantaneous motion of the link $B_{i} C_{i}$ is translational. The wrench $\mathbf{R}_{i}\left(\begin{array}{llllll}r_{i x} & r_{i y} & 0 & 0 & 0 & r_{i z}^{0}\end{array}\right)$ $(i=1,2,3)$ can be determined using the equation analogous to (1). The pressure angle can be found using the equation (3).

Thus, in each position we determine $m$ pressure angles corresponding to all $m$ degrees of freedom. Then we consider the maximum value of these angles. By such a way, we can determine the pressure angles corresponding to the different structures distinguished by different input links and obtain all possible workspace with singularity-free zones. It is examined in the next section. 


\section{Numerical plotting of singularity-free zones taking account of pressure angle}

In this section we would like to show the singularity-free zones in the workspace of 3RPR parallel manipulator with modified legs. These zones have been determined by using the maximum values of the pressure angles.

For numerical simulation we consider 3-RPR parallel manipulator in which the basic triangle $A_{1} A_{2} A_{3}$ is equilateral with radius $0.35 \mathrm{~m}$ (Fig. 1) and the platform also represents an equilateral triangle with radius $0.1 \mathrm{~m}$. In other words the centers of the joints mounted on the frame are disposed on the circle with radius $0.1 \mathrm{~m}$. The rotation of the revolute joints $A_{i}$ is limited to $\pm 90^{\circ}$ respect to the orientation of links $A_{i} D_{i}$ with the mechanism in the central symmetric configuration. For added dyads $A_{i} D_{i}=C_{i} D_{i}=0.25 \mathrm{~m}$, the articulated dyads are always located on the left of the prismatic pairs as it is shown in Figure 3 and the translation of the prismatic pairs are limited relative to the joints $A_{i}$ and $C_{i}$ by values $\left(A_{i} C_{i}\right)_{\min }=\left(B_{i} C_{i}\right)_{\min }=0.05 m$.

Taking into account that the manipulator can be actuated either by links $A_{i} D_{i}$ or by links $A_{i} B_{i}$, for given output parameters $\mathbf{x}=(x, y, \phi)^{\mathrm{T}}$ of the platform, we have 8 different combinations of actuation, i.e. we have 8 different combinations of input parameters presented below (underlined letters show the input pairs, "R" for input links $A_{i} B_{i}$ with input angles $\theta_{i}$ and "P" for input links $A_{i} D_{i}$ with input displacements $\rho_{i}$ ):

$$
\begin{aligned}
& \text { RRR : } \underline{\text { RPR- RPR- }} \text { RPR : } \mathbf{q}_{(1)}=\left(\theta_{1}, \theta_{2}, \theta_{3}\right)^{\mathrm{T}} \\
& \text { RRP : } \underline{\text { RPR }}-\underline{\mathrm{RPR}}-\mathrm{R} \underline{\mathrm{PR}}: \mathbf{q}_{(2)}=\left(\theta_{\mathbf{1}}, \theta_{2}, \rho_{3}\right)^{\mathrm{T}}
\end{aligned}
$$

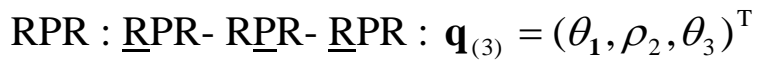

$$
\begin{aligned}
& \text { RPP : RPR- RPR-RPR : } \mathbf{q}_{(4)}=\left(\theta_{1}, \rho_{2}, \rho_{3}\right)^{\mathrm{T}} \\
& \text { PRR : RPR- RPR- } \underline{\mathrm{RPR}}: \mathbf{q}_{(5)}=\left(p_{1}, \theta_{2}, \theta_{3}\right)^{\mathrm{T}} \\
& \text { PRP : RPR- RPR-RPR : } \mathbf{q}_{(6)}=\left(\rho_{1}, \theta_{2}, \rho_{3}\right)^{\mathrm{T}} \\
& \text { PPR : R } \underline{P R}-\mathrm{R} \underline{P R}-\mathrm{RP} \underline{\mathrm{R}}: \mathbf{q}_{(7)}=\left(p_{1}, \rho_{2}, \theta_{3}\right)^{\mathrm{T}} \\
& \text { PPP : RPR-RPR-RPR : } \mathbf{q}_{(8)}=\left(p_{1}, \rho_{2}, \rho_{3}\right)^{\mathrm{T}}
\end{aligned}
$$

Tables 1 and 2 show the workspaces of each case of actuation with $0^{\circ}$ and $45^{\circ}$ orientation angles (the origin of the fixed base frame is located at the centre of the equilateral triangle $A_{1} A_{2} A_{3}$ ). In these figures, several zones can be seen, which correspond to the variations of the maximum values of the pressure angle for given position of the platform. The contrast intensity shows the variations of the pressure angle (see Figure 4).

$\square$ pressure angle $<15 \mathrm{deg}$
$\square \quad$ pressure angle between $15 \mathrm{deg}$ and $30 \mathrm{deg}$
pressure angle between $30 \mathrm{deg}$ and $45 \mathrm{deg}$
$\square$ pressure angle between $45 \mathrm{deg}$ and $60 \mathrm{deg}$
pressure angle between $60 \mathrm{deg}$ and $75 \mathrm{deg}$
pressure angle $>75 \mathrm{deg}$

Fig. 4. The contrast intensity corresponding to the pressure angle. 
Thus, the black zones are the surfaces where the pressure angle has inadmissible values, and as a result, these are the zones, which cannot be reached by the parallel mechanism. 
Table 1

Maximum values of the pressure angles $\left(\phi=0^{\circ}\right)$

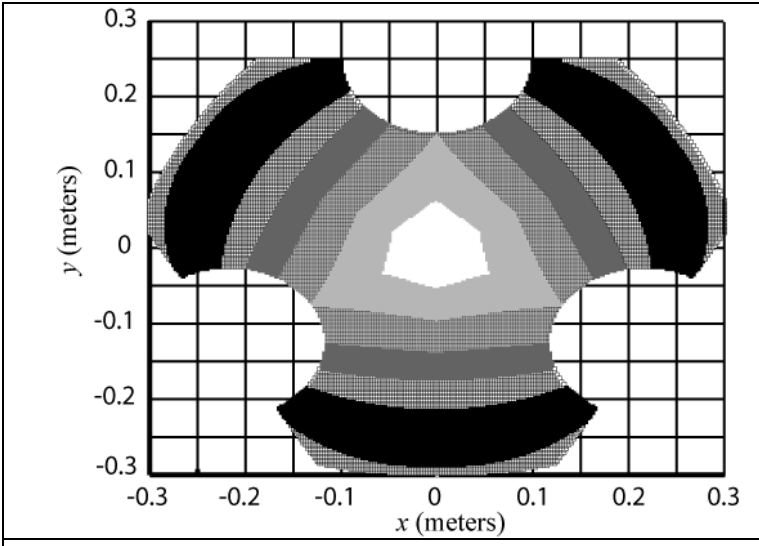

(a) Actuators: RRR

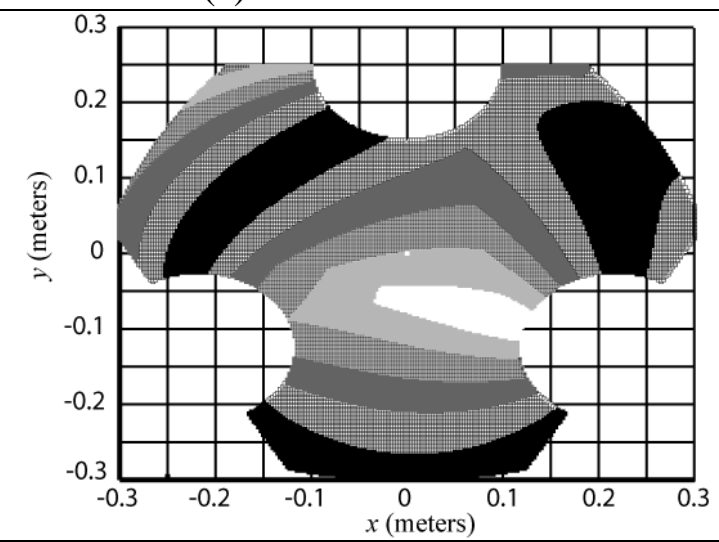

(c) Actuators: PRR

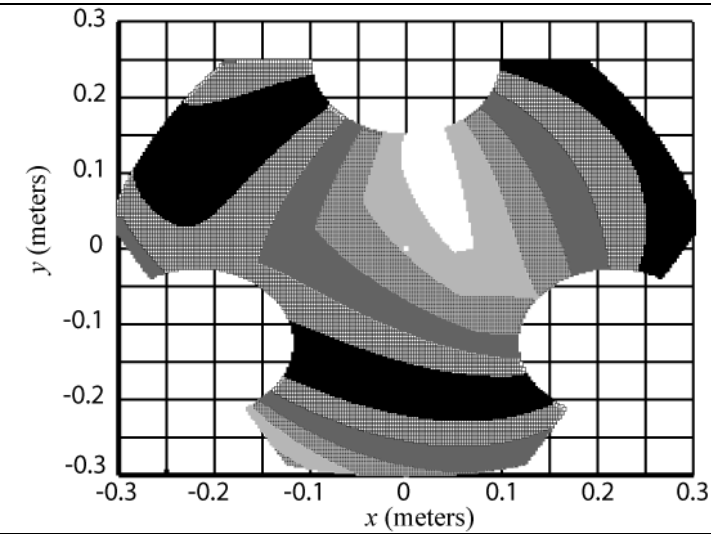

(e) Actuators: RPR

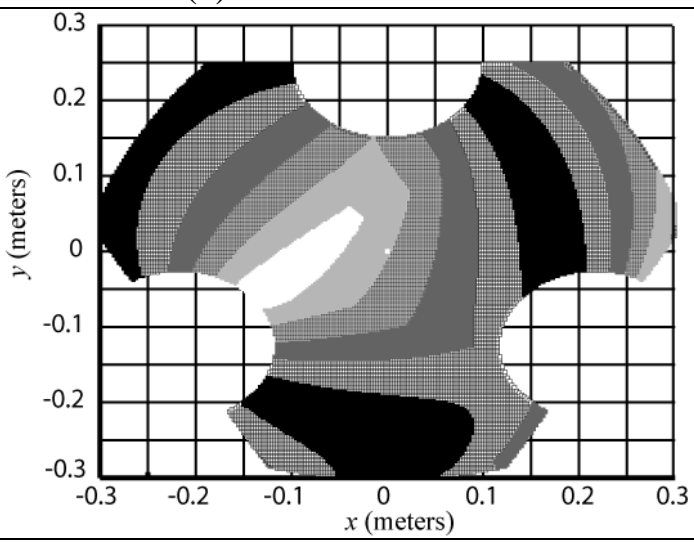

(g) Actuators: RRP

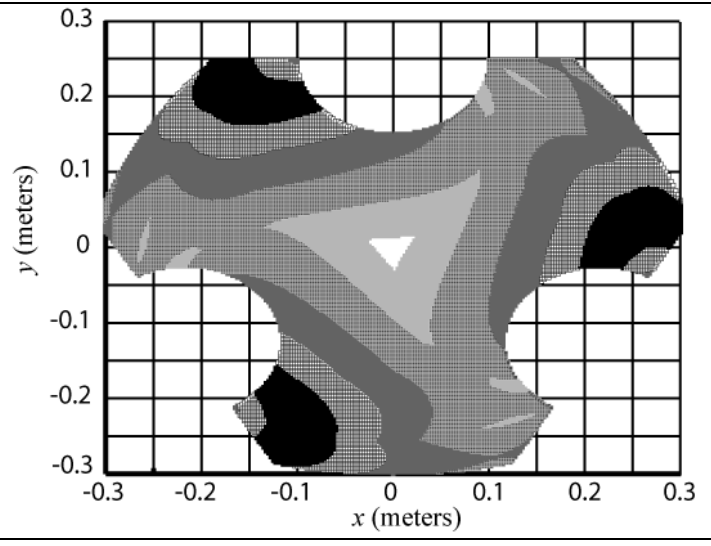

(b) Actuators: PPP

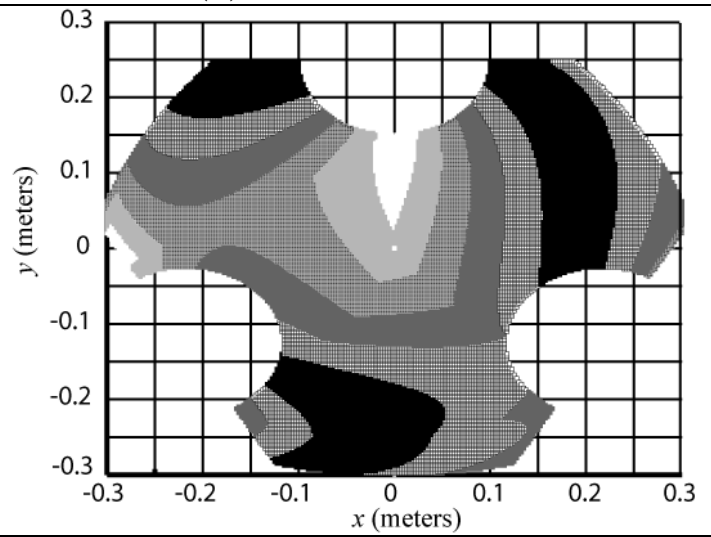

(d) Actuators: RPP

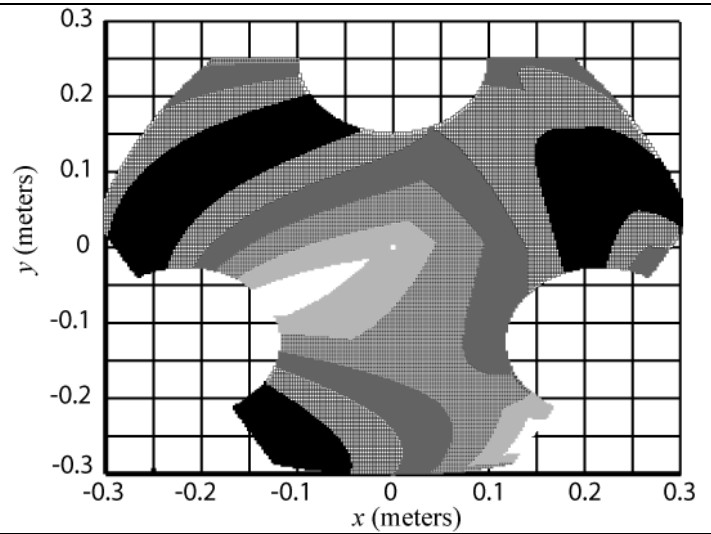

(f) Actuators: PRP

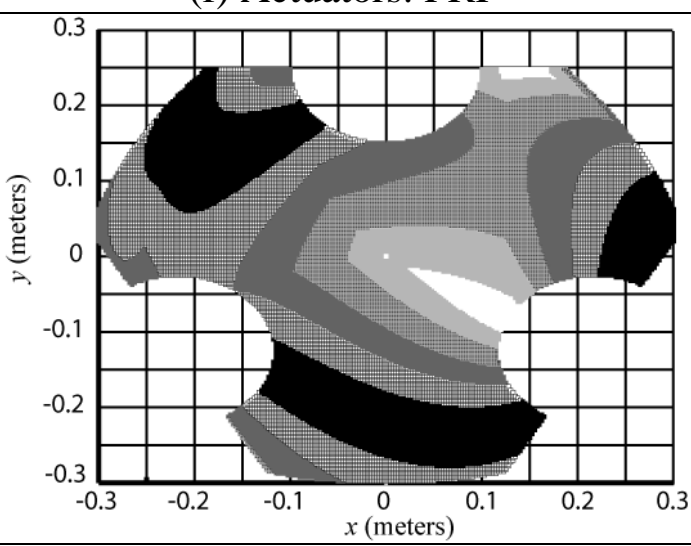

(h) Actuators: PPR 
Table 2

Maximum values of the pressure angles $\left(\phi=45^{\circ}\right)$

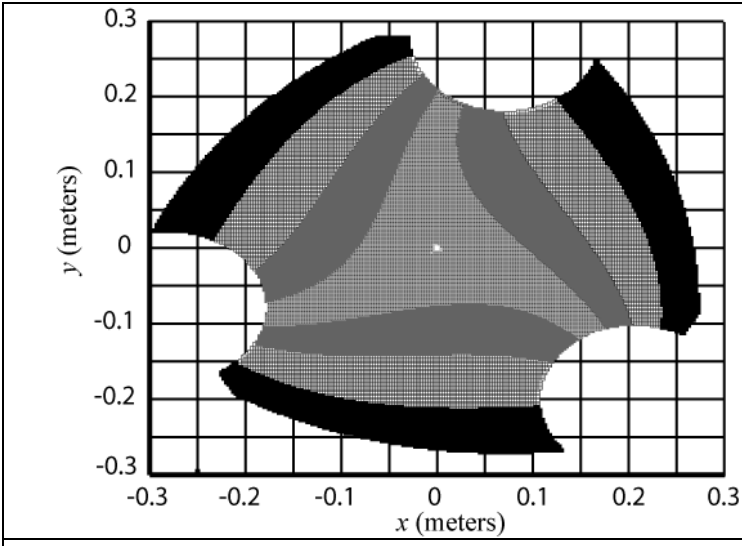

(a) Actuators: RRR

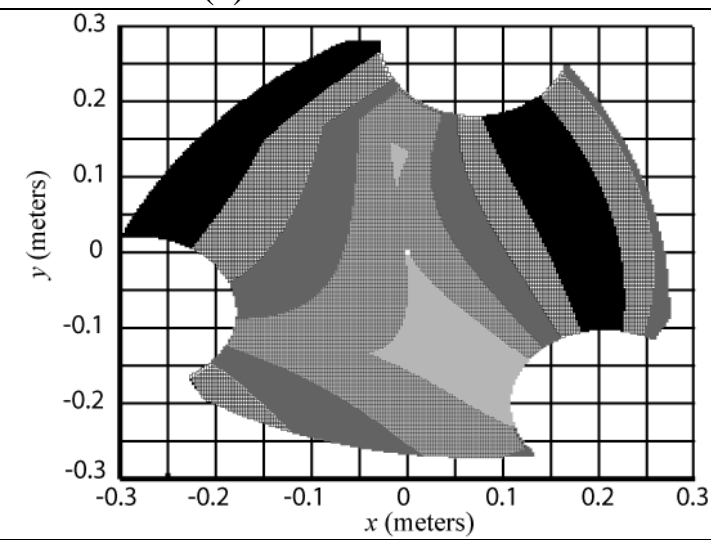

(c) Actuators: PRR

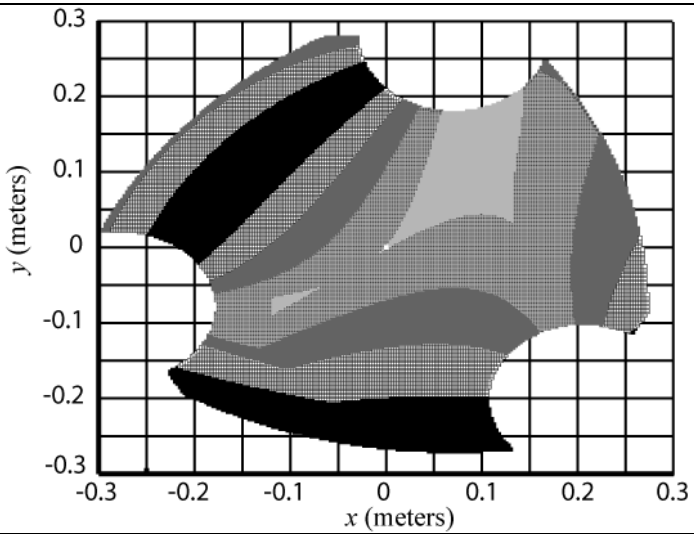

(e) Actuators: RPR

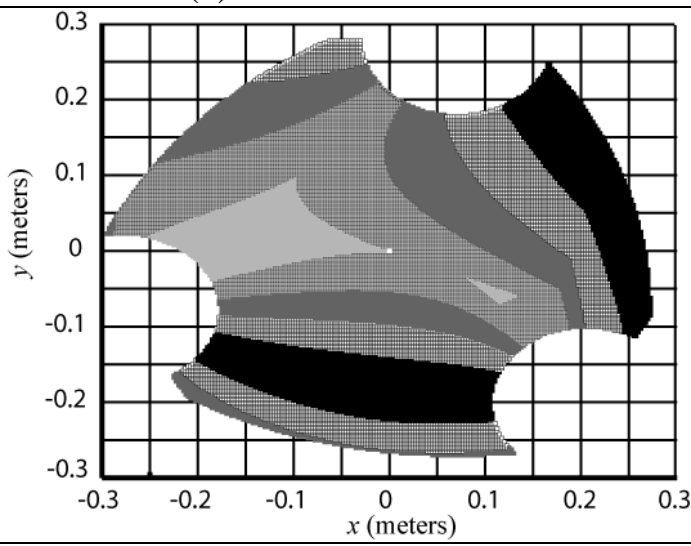

(g) Actuators: RRP

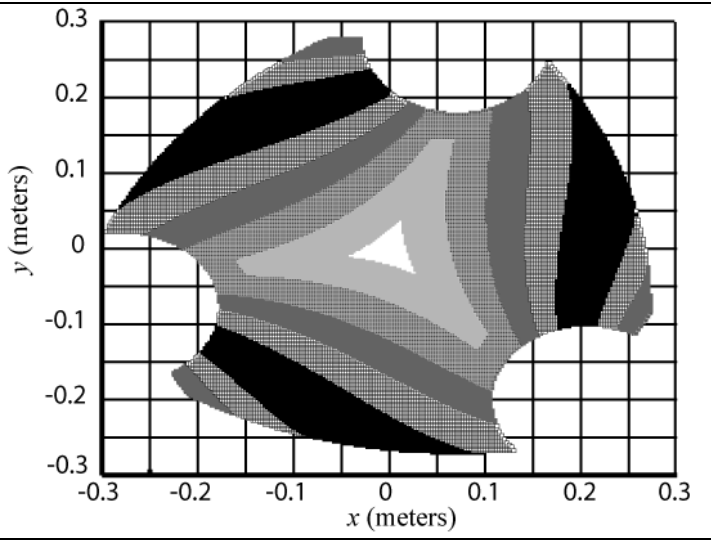

(b) Actuators: PPP

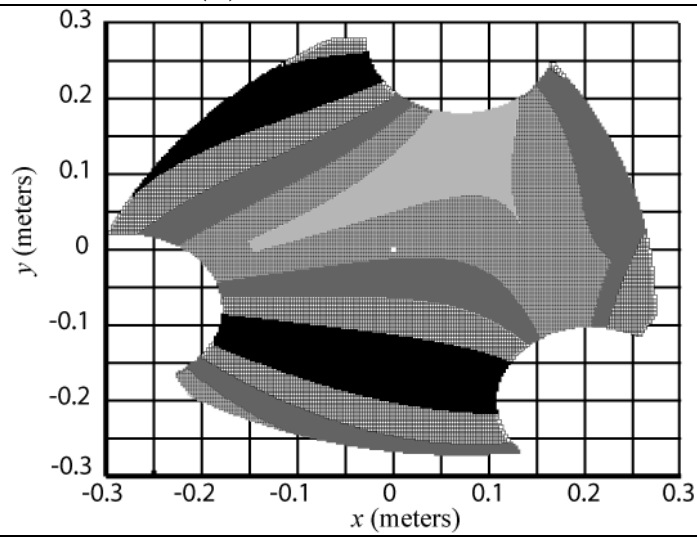

(d) Actuators: RPP

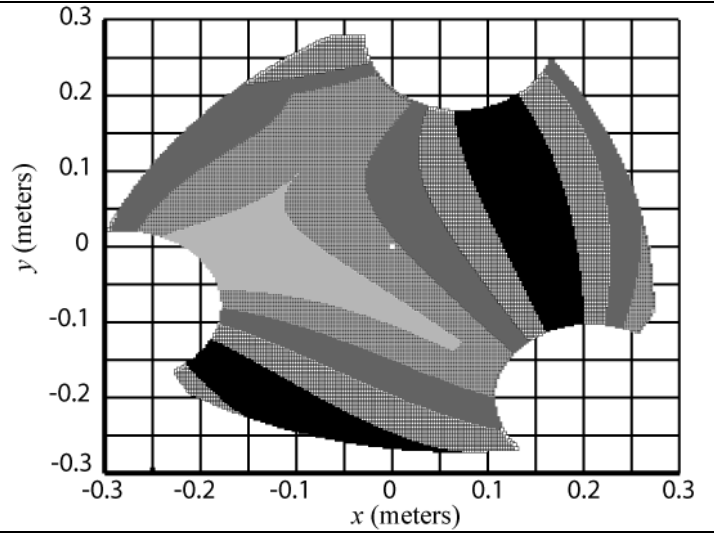

(f) Actuators: PRP

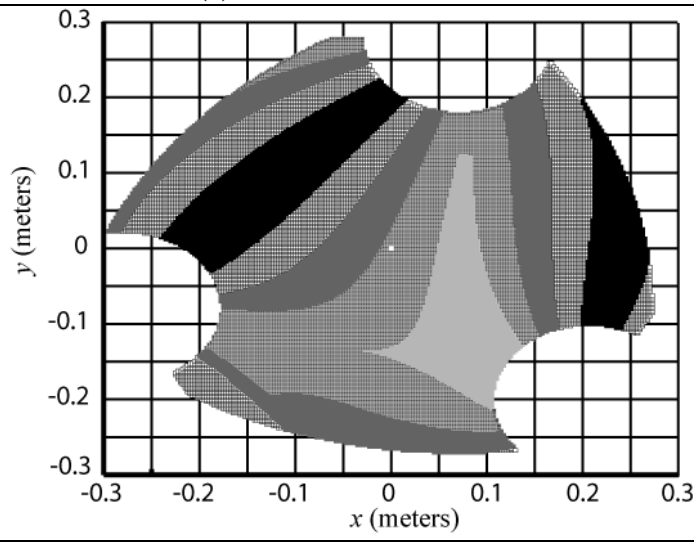

(h) Actuators: PPR 
The table 3 shows the ratio between the total value of singularity-free volumes and the total workspace for each case of actuation (for two examined cases: $\phi=0^{\circ}$ and $\phi=45^{\circ}$ ).

Table 3

Total value of singularity-free volumes for each case of actuation

\begin{tabular}{|l|c|c|c|c|}
\hline \multirow{2}{*}{$\begin{array}{l}\text { Type of } \\
\text { actuatio } \\
\mathrm{n}\end{array}$} & $\begin{array}{c}\left.\phi=0 \mathrm{deg} \text { (workspace surface: } 0.21 \mathrm{~m}^{2}\right) \\
\text { Singularity-free } \\
\text { zones }\left(\mathrm{m}^{2}\right)\end{array}$ & $\begin{array}{c}\text { Singularity-free } \\
\text { zones relative to } \\
\text { the whole } \\
\text { workspace }\end{array}$ & $\begin{array}{c}\text { Singularity-free } \\
\text { zones }\left(\mathrm{m}^{2}\right)\end{array}$ & $\begin{array}{c}\text { Singularity-free } \\
\text { zones relative to the } \\
\text { whole workspace }\end{array}$ \\
\hline RRR & 0.137 & $65 \%$ & 0.147 & $74 \%$ \\
\hline PPP & 0.181 & $86 \%$ & 0.152 & $76 \%$ \\
\hline PRR & 0.152 & $72 \%$ & 0.158 & $79 \%$ \\
\hline RPR & 0.152 & $72 \%$ & 0.158 & $79 \%$ \\
\hline RRP & 0.152 & $72 \%$ & 0.158 & $79 \%$ \\
\hline RPP & 0.155 & $74 \%$ & 0.165 & $83 \%$ \\
\hline PRP & 0.155 & $74 \%$ & 0.165 & $83 \%$ \\
\hline PPR & 0.155 & $74 \%$ & 0.165 & $83 \%$ \\
\hline
\end{tabular}

Figure 5 shows the reachable workspace of the modified parallel mechanism with legs of variable structure. We can see that the workspace of the modified manipulator is only composed of singularity-free zones and the whole workspace of the manipulator is reachable (increase until 100\%).

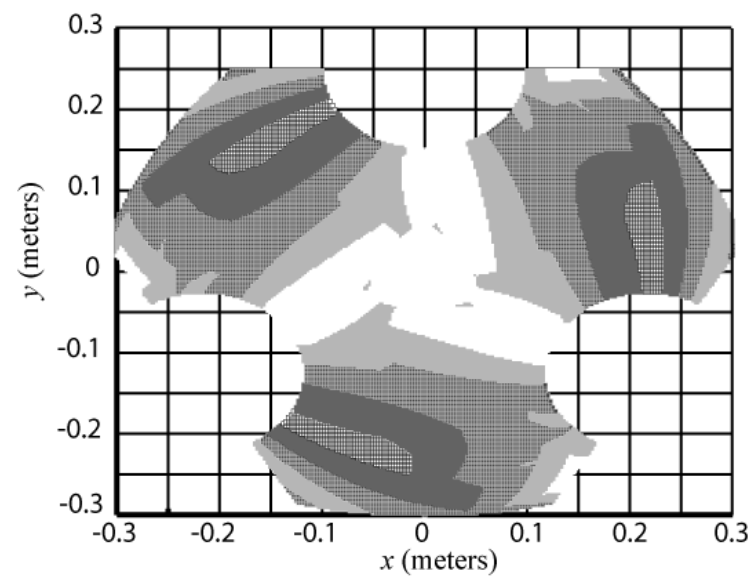

(a) $\phi=0 \mathrm{deg}$

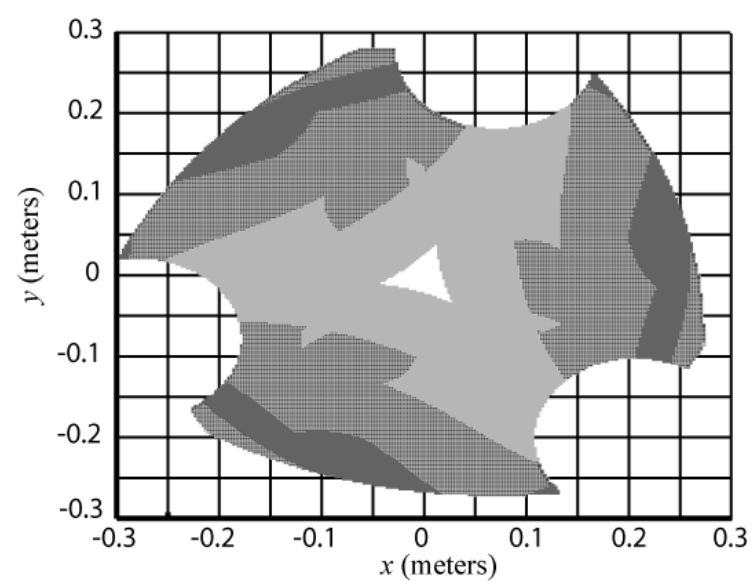

(b) $f=45 \mathrm{deg}$

Fig. 5. The reachable workspace of the parallel manipulator with modified legs.

\section{Trajectory planning and design procedure for determination of optimal leg structure}

In order to obtain the best structural architecture of the manipulator for a given trajectory, in this section we describe a procedure, which allows determining the optimal system of actuation. This algorithm is based on the control of the pressure angles in the joints of the manipulator along the given trajectory (Fig. 6). 


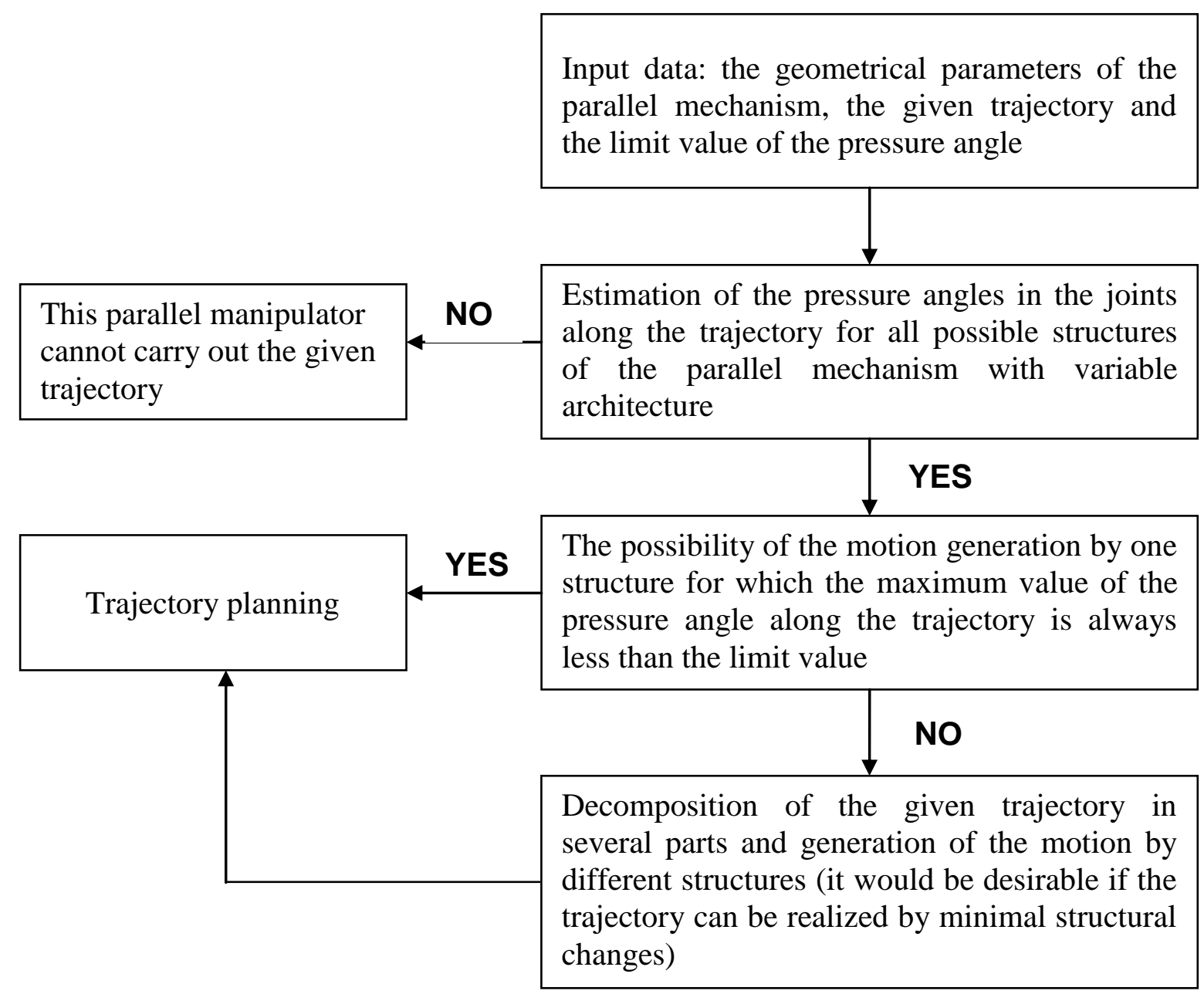

Fig. 6. Procedure to determination of the optimal structure of the parallel manipulator taking into account the limit pressure angle.

Two numerical examples are considered below in order to illustrate the application of the suggested design procedure.

Problem No. 1. For given parallel manipulator (Fig. 1) with legs of variable structure (Fig. 3) generate the trajectory by straight line from the initial position $P_{1}\left(x_{1}=0, y_{1}=0, \phi_{1}=0\right)$ to the final position $P_{2}\left(x_{2}=-0.25 m, y_{2}=0, \phi_{2}=0\right)$.

The estimation of the pressure angle along the given trajectory shows that the best structural solution for generation of motion is the $\underline{R P R}-\mathrm{R} P \mathrm{PR}-\mathrm{R} \underline{\mathrm{PR}}$ mechanism, i.e. when the first actuator is connected with the link $A_{1} C_{1}$ and two others with the links $A_{2} D_{2}$ and $A_{3} D_{3}$. In this case the maximum values of the pressure angles in the joints are always less than the limit value.

In order to illustrate the variations of torques for examined case we develop a model of the manipulator with the given trajectory using the ADAMS software. A force parallel to the $\boldsymbol{x}$-axis and equal to $100 \mathrm{~N}$ was applied to the platform and the friction coefficients in the prismatic pairs were equal to 0.01. The obtained torques are shown in Figure 7. We can note that the torques have admissible values along the trajectory. 


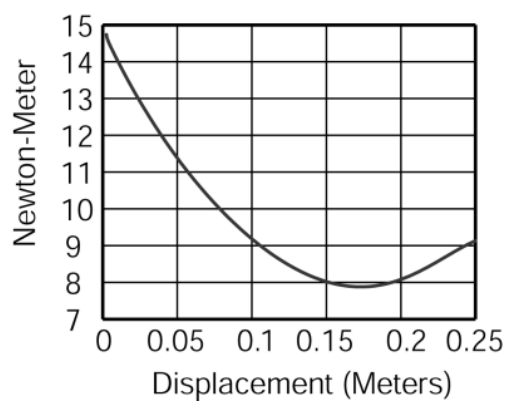

(a) Actuator 1

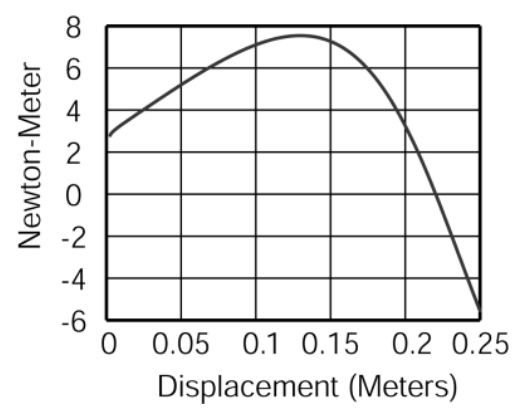

(a) Actuator 2

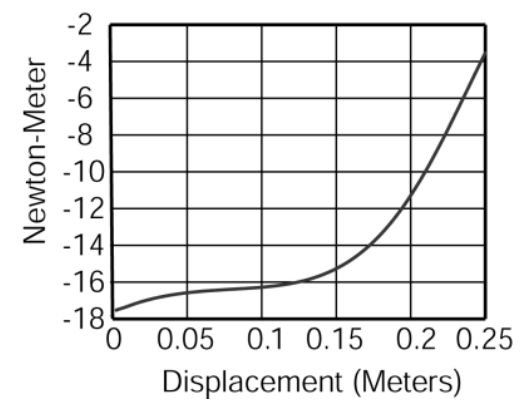

(a) Actuator 3

Fig. 7. Torques of the actuators.

Problem No. 2. For given parallel manipulator (Fig. 1) with legs of variable structure (Fig. 3) generate the trajectory by straight lines from the initial position $P_{1}\left(x_{1}=0, y_{1}=0, \phi_{1}=0\right)$ to the second position $P_{2}\left(x_{2}=0.1 \mathrm{~m}, y_{2}=-0.25 \mathrm{~m}, \phi_{2}=0\right)$ and then to the final position $P_{3}\left(x_{3}=-0.1 m, y_{3}=-0.25 m, \phi_{3}=0\right)$.

In this case, the estimation of pressure angle shows that it is impossible to carry out the given trajectory by one structural system. In a first time, the trajectory from initial position $P_{1}\left(x_{1}=0, y_{1}=0, \phi_{1}=0\right)$ to the second position $P_{2}\left(x_{2}=0.1 \mathrm{~m}, y_{2}=-0.25 m, \phi_{2}=0\right)$ must be carried out by the RPR- $\underline{R} P R-R \underline{P R}$ mechanism then from the second position $P_{2}\left(x_{2}=0.1 m, y_{2}=-0.25 m, \phi_{2}=0\right)$ to the final position $P_{3}\left(x_{3}=-0.1 m, y_{3}=-0.25 m, \phi_{3}=0\right)$ by the RPR-RPR-RPR mechanism. Thus the suggested solution based on these structural architectures allows obtaining the optimal actuation system of the manipulator considering the pressure angle.

The obtained torques are shown in Figure 8. We can note that the torques have admissible values along the trajectory but there is a discontinuity in the point $P_{2}$ caused by the structural change of the parallel mechanism.

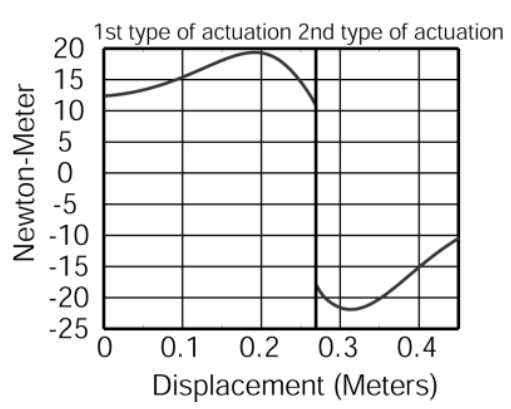

(a) Actuator 1

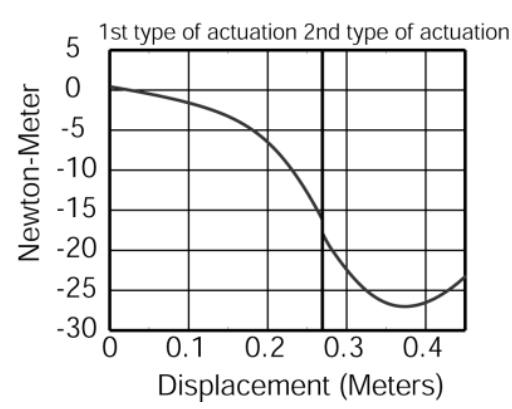

(a) Actuator 2

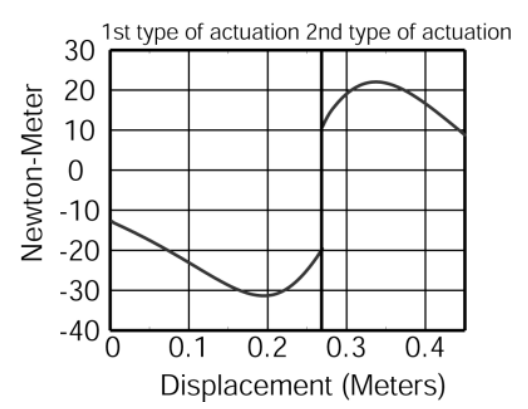

(a) Actuator 3

Fig. 8. Torques of the actuators.

It should be noted that the mechanism of variable structure shown above was developed by means of the added articulated dyads, but, it is obvious that such a mechanism can be designed on the base of the screw or cam systems, the rhombic pantographs, etc.

In a similar way one obtains the increase of singularity-free zones in the workspace of parallel manipulators only with revolute pares. In Fig. 9 is illustrated a 3-RRR parallel manipulator with the legs of variable structure. 


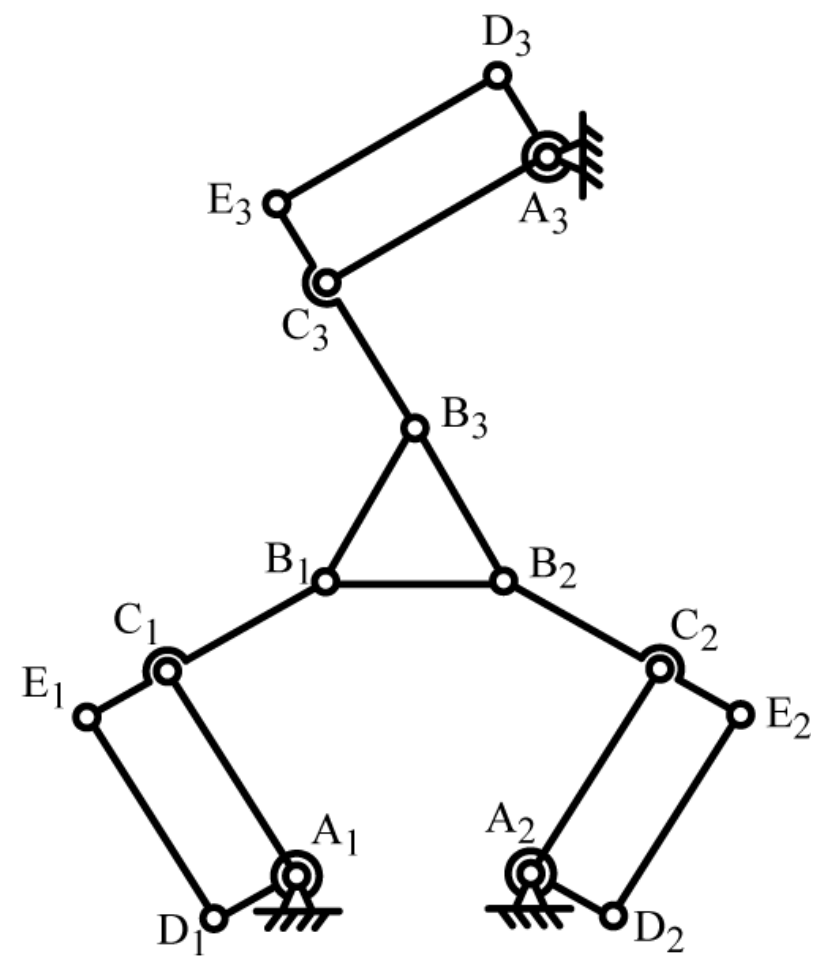

Fig. 9. Planar parallel manipulator 3-RRR with legs of variable structure.

The rotating actuators are mounted on the base and connected by electromagnetic clutches with the links $A_{i} C_{i}$ and $A_{i} D_{i}$. These two input links cannot actuated simultaneously and the input motion can be transmitted either by the link $A_{i} C_{i}$ or $A_{i} D_{i}(i=1,2,3)$. In this way we can obtain the leg's mechanisms with different structural parameters and carry out the given trajectory taking into account the limit value of the pressure angle. We shall not treat the procedure of resolution that it differs from the previous case only by determination of the pressure angle.

\section{Conclusions}

A procedure for the increase of singularity-free zones in the workspace of planar parallel manipulators has been presented in this paper. The procedure is based on the known kinematic singularity equations and the control of the pressure angles in the joints of the manipulator along the given trajectory of the platform. The zones, which cannot be reached by the manipulator, were detected. For increase of the reachable workspace of the manipulator the legs of variable structure were proposed. Such a solution allows obtaining the best structural architecture of the manipulator for any trajectory. The design of the optimal structure of the planar parallel manipulator 3-RPR was illustrated by two numerical simulations. We believe that the suggested method is a useful tool for the improvement of the functional performance of parallel manipulators with singular zones.

Finally, it should be noted that the same problem for spatial parallel manipulators was studied in [26].

\section{Acknowledgement}

The authors thank Philippe Wenger and Damien Chablat (IRCCyN of Nantes) for very useful discussions while editing of the manuscript. 


\section{References}

[1] J. P. Merlet. Parallel robots. Kluwer Academic Publishers (2000) 372p.

[2] V. Glazunov, A. Kraynev, R. Bykov, G. Rashoyan, N Novikova. Parallel manipulator control while intersecting singular zones. Proceedings of the 15th Symposium on Theory and Practice of Robots and Manipulators (RoManSy) CISM-IFToMM, Montreal (2004).

[3] K. Alvan, A. Slousch. On the control of the spatial parallel manipulators with several degrees of freedom. Mechanism and Machine Theory, Saint-Petersburg, ${ }^{\circ} 1$ (2003) pp. $63-69$.

[4] C.M. Gosselin, J. Angeles. Singularity analysis of closed-loop kinematic chains. IEEE Transactions on Robotics and Automatics. 6(3) (1990) pp.281-290.

[5] O. Ma, J. Angeles. Architecture singularities of parallel manipulators. The International Journal of Robotics and Automation, 7(1) (1992) pp.23-29.

[6] D. Zlatanov, R.G. Fenton and B. Benhabib. Singularity analysis of mechanisms and robots via a velocity-equation model of the instantaneous kinematics. Proceedings of the 1994 IEEE International Conference on Robotics and Automation, 2 (1994) pp. 980-991.

[7] J.-P. Merlet. Singular configurations of parallel manipulators and Grassmann geometry. The international Journal of Robotics Research. 8(5) (1989) pp. 45-56.

[8] B.M. St-Onge, C.M. Gosselin. Singularity analysis and representation of the general Gough-Stewart platform. The International Journal of Robotics Research. 19(3) (2000) pp.271-288.

[9] F. Pernkopf, M. Husty. Singularity analysis of spatial Stewart-Gough platforms with planar base and platform. Proceedings of the DECT 2002, Montreal (2002).

[10]J.T. Wen, J.F. Oapos Brien. Singularities in three-legged platform-type parallel mechanisms. IEEE Transactions on Robotics and Automation. 19(4) (2003) pp.720- 726.

[11]S. Bandyopadhyay, A. Ghosal. Analysis of configuration space singularities of closedloop mechanisms and parallel manipulators. Mechanism and Machine Theory, 39 (5) (2004) pp.519-544.

[12] J.-S. Zhao, Z.-J. Feng, K. Zhou and J.-X. Dong. Analysis of the singularity of spatial parallel manipulator with terminal constraints. Mechanism and Machine Theory, 40(3) (2005) pp.275-284.

[13]P. Ben-Horin and M. Shoham. Singularity analysis of a class of parallel robots based on Grassmann-Cayley algebra. Mechanism and Machine Theory, Vol. 41 (8), (2006), pp. 958-970.

[14]Haidong Li, Clément M. Gosselin and Marc J. Richard. Determination of maximal singularity-free zones in the workspace of planar three-degree-of-freedom parallel mechanisms. Mechanism and Machine Theory, Vol. 41(10) (2006), pp. 1157-1167.

[15] Glazunov V.A. Koliskor A.Sh., Krainev A.F., and Model B.I. Classification principles and analysis methods for parallel-structure spatial mechanisms. / Journal of Machinery Manufacture and Reliability, Allerton Press Inc., 1990, No 1, p. 30-37.

[16] V. Glazunov. Twists of movements of parallel mechanisms inside their singularities. Mechanism and Machine Theory, Vol. 41 (10), (2006), pp. 1185-1195.

[17] S. Balli, S. Chand. Transmission angle in mechanisms. Mechanism and Machine Theory, 37 (2002) pp. 175-195.

[18] G. Sutherland, B. Roth. A transmission index for spatial mechanisms. Transactions of the ASME. Journal of Engineering for Industry. (1973) pp. 589-597.

[19]G. Sutherland. Quality of motion and force transmission. Mechanism and Machine Theory. 16(3) 191 p. 221-225.

[20]C.-C. Lin, W.-T. Chang. The force transmissivity index of planar linkage mechanisms. Mechanism and Machine Theory, 37 (2002) p. 1465-1485. 
[21]C. Chen, J. Angeles. A generalized transmission index for spatial linkages. Proceedings of the ASME 2005 IDETC/CIE Conference, September 24-28, Long Beach, California (2005).

[22] O. Alba-Gomez, P. Wenger, and A. Pamanes. Consistent kinetostatic indices for planar 3DOF parallel manipulators, application to the optimal kinematic inversion. Proceedings of the ASME 2005 IDETC/CIE Conference, September 24-28, Long Beach, California (2005).

[23]Lee, J., Duffy, J., Keler, J., "The Optimum Quality Index for the Stability of In Parallel Planar Platform Devices, Trans. ASME, Journal of Mechanical Design, Vol. 121, March, 1999, pp. 15-20.

[24]F.M. Dimentberg. The screw calculus and its applications in mechanics. Moscow, (1965) 327p. (English translation: AD680993, Clearinghouse for Federal Technical and Scientific Information, Virginia).

[25]I.A. Bonev, D. Zlatanov, C.M. Gosselin. Singularity analysis of 3-DOF planar parallel mechanisms via Screw Theory. Transactions of the ASME. Journal of Mechanical Design. 2003, Vol. 125, pp. 573-581.

[26] V. Arakelian, S. Briot and V. Glazunov. Improvement of Functional Performance of Spatial Parallel Manipulators Using Mechanisms of Variable Structure. In the Proceedings of the 12th World Congress in Mechanism and Machine Science, June 1821, 2007, Besançon, France. 